\title{
Patient Satisfaction and Symptoms Improvement in Women Using a Vginal Pessary for The Treatment of Pelvic Organ Prolapse
}

\author{
Nahid Radnia ${ }^{1}$, Maryam Hajhashemi², Tahereh Eftekhar ${ }^{3}$, Maryam Deldar ${ }^{3}$, Taraneh Mohajeri ${ }^{4}$, Samira Sohbati ${ }^{5}$, Zinat Ghanbari ${ }^{3}$ \\ 1. Department of Obstetrics \& Gynecology, Faculty of Medicine, Hamadan University of Medical Sciences, Hamadan, Iran \\ 2. Department of Obstetrics \& Gynecology, Faculty of Medicine, Isfahan University of Medical Sciences, Isfahan, Iran \\ 3. Department of Obstetrics \& Gynecology, Vali-e-Asr Hospital, Tehran University of Medical Sciences, Tehran, Iran \\ 4. Department of Obstetrics \& Gynecology, Mashhad Islamic Azad University of Medical Sciences, Mashhad, Iran \\ 5. Department of Obstetrics \& Gynecology, Kerman University of Medical Sciences, Kerman, Iran
}

\author{
Corresponding Author: \\ Zinat Ghanbari \\ Department of Obstetrics \& Gynecology, Vali-e-Asr Hospital, \\ Tehran University of Medical Sciences, Tehran, Iran \\ Nahid Radnia \\ Department of Obstetrics \& Gynecology, Faculty of Medicine, \\ Hamadan University of Medical Sciences, Hamadan, Iran. \\ Tel.: 09121305814 Email: drz_ghanbari@yahoo.com \\ Received: May 9th, 2019 - Accepted: August 9th, 2019
}

\begin{abstract}
Pelvic organ prolapse is a common complaint among older women. Vaginal pessary insertion is an appropriate treatment as a non-surgical method with few complications. This paper is a prospective observational study of 68 patients with pelvic organ prolapse that was carried out at the Imam Khomeini Hospital's Pelvic Floor clinic. The degree of pelvic organ prolapse was graded according to the Pelvic Organ Prolapse Quantification (POP-Q) System. For all patients, the Pelvic Floor Distress Inventory-20 (PFDI-20) questionnaire was completed before vaginal pessary insertion, and after approximately 6 months of treatment. After 6-8 months, we found out that vaginal discharge was significantly increased and the feeling of fullness in the vagina was significantly decreased. However, sexual dissatisfaction, the feeling of incomplete evacuation, fecal and urinary incontinence, frequent urination, and pain or discomfort in the genital region were not significantly different after using a pessary. Approximately half a year later, $96.7 \%$ of the women with a successful pessary fitting trial were satisfied and reported a significant improvement in symptoms. Further studies with larger sample size, a different type of pessary, and a longer follow-up duration are recommended to evaluate all the symptoms associated with pelvic organ prolapse and its treatment.
\end{abstract}

Keywords: Pessaries, Pelvic Floor, Pelvic Organ Prolapse

\section{Introduction}

Pelvic organ prolapse (POP) is the herniation of the pelvic organs to or beyond the vaginal walls. Most women with POP experience symptoms that impact their quality of life such as sexual function, daily activities and exercise [1].

The pelvic structures that may be involved in pelvic organ prolapse include the uterus (uterine prolapse) or vaginal apex (apical vaginal prolapse), anterior vaginal wall (cystocele), or posterior vaginal wall (rectocele).

Anatomic support of the pelvic organs in women is provided by an interaction between the muscles of the pelvic floor, like the levator ani muscle complex, and connective tissue attachments within the bony pelvis. All levels of vaginal support are connected through a continuous endopelvic fascia support network; these structures stabilize the pelvic organs in the correct position $[2,3]$.
Pelvic organ prolapse may be symptomatic or asymptomatic. Proven risk factors for POP include parity, advanced age, chronic constipation and obesity $[4,5]$. Treatment methods for pelvic organ prolapse have not been completely studied. There are two basic patterns for treating POP, including conservative treatment and surgery. Surgical repair is one approach to POP treatment. However, some women may prefer to avoid surgical treatment or may not be candidates for surgery [21]. Vaginal pessaries are intravaginal support devices that may be controlled by the patient. These devices reduce prolapse or incontinence [7] and are an alternative non-invasive treatment option with rapid symptom relief without surgical complications for women with these conditions. Pessary use is the most cost-effective treatment alternative for treating POP [22]. Some evidence suggests that women with POP who use vaginal pessary have a lower stage of prolapse on 
clinical examinations after pessary insertion [8]. Patient's acceptance of pessaries varies from 42 to 100 percent [9-12] and is related to appropriate counseling and encouragement from the physician [13]. In this study, we evaluated the satisfaction and symptom improvement of patients using a vaginal pessary in order to treat POP.

\section{Materials and Methods}

Women with symptomatic pelvic organs prolapse referred to the Pelvic Floor clinic at Imam Khomeini Hospital, and they were advised to use a vaginal pessary, the physician describing the advantages and disadvantages to the patients.

The 68 patients with pelvic organ prolapse, who met the inclusion criteria (pelvic organ prolapses with $P O P-Q$ stage $\geq 2$ in one or more components, patients who preferred conservative therapy to surgery and patients who had no active pelvic infection) and agreed to try a vaginal pessary were included in the study. After the patients underwent pessary insertion, long-term follow-up was carried out. Also, vaginal estrogen was prescribed for all patients included in this study.

At the first visit, demographic data were collected, and all patients were examined. The degree of pelvic organ prolapse was graded according to the $P O P-Q$ test. For all patients, the Pelvic Floor Distress Inventory-20 (PFDI-20) valid questionnaire was completed during the first visit and follow-up.

Approximately 6 months (from 6 to 8 months) after pessary insertion, the patients came back to the clinic, and the POP-Q test was performed. The Patient Global Impression of Improvement (PGI-I) valid questionnaire was used to assess patient satisfaction after pessary insertion.

PGI-I is a single question and global index transition scale that is asking the patient to rate their urinary tract condition and the response of a condition to a therapy (transition scale). It is simple, direct, yet easy to use and intuitively understandable to clinicians. Additionally, PGI-I has excellent construct validity [14].

There are different types of vaginal pessaries that we used for different patients as needed (ring with support, Gelhorn and cube pessary). In all cases, we used a type of pessary that was appropriate to the patient's needs and associated conditions.

During follow-up examination, pessary placement was first checked after 1 or 2 weeks. If the patient was comfortable and satisfied with the pessary, a next follow-up visit was scheduled at 6 months.

All patients completed the PGI-I questionnaire again during the follow-up visit in the Pelvic Floor clinic before the examination and removal of the pessary. The average 6-month responses were compared with the baseline responses.

T-test was used to compare the means in normal data while the Mann-Whitney U test was used to analyze abnormal data. Statistical analysis was carried out using SPSS 20. P values of $<0.05$ were considered statistically significant

\section{Results}

Of the 68 patients with symptomatic pelvic organ prolapse (stage $\geq 2 \mathrm{POP}-\mathrm{Q}$ ) with a mean age of 68.9 years (ranging from 34 to 89 years) who met the inclusion criteria, 60 patients $(88 \%)$ successfully retained the pessary approximately 6 months after insertion. Two patients could not retain the pessary and six patients left the study and underwent surgery.

In our study, six (9\%) patients had a history of prolapse surgery, six (9\%) patients had a history of hysterectomy, 29 (42.6\%) patients were sexually active, $8(12.1 \%)$ patients tended to undergo surgery at the first visit and 66 (97.1\%) patients were postmenopausal Table1.

At the first follow-up visit, $30 \%$ of patients reported significant vaginal discharge and cervical erosion after pessary insertion $(P<0.001)$, which improved relatively at the 8-month follow up.

At the first visit, the PDFI-20 questionnaire was completed by all patients. 34 patients complained of pelvic organ prolapse and 10 patients complained of colorectal and anal symptoms. 17 patients had a feeling of fullness in the vagina, 25 patients experienced frequent urination, and 18 patients experienced urgency, some patients had urine leakage(as wet urgency) and some patients didn't have leakage until urination occure (dry urgency).

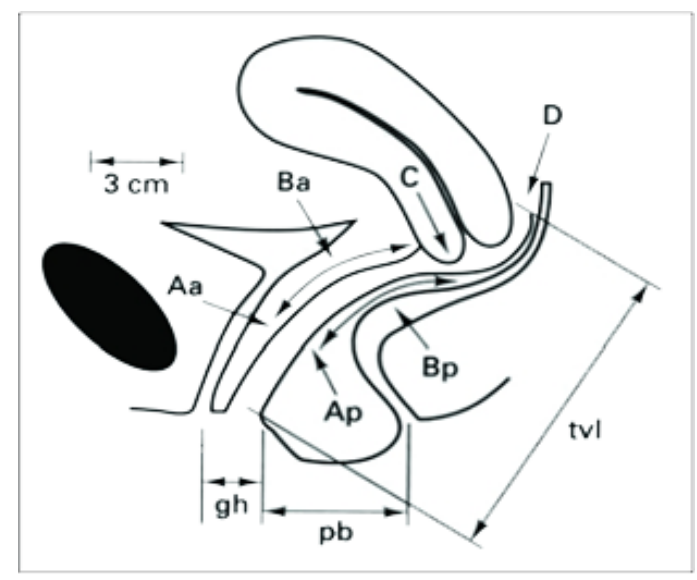

I have tendency to use pessary and enter the study.

Signature:

Name \& Family:

Date:

Figure 1: POP-Q points 
In order to determine POP-Q status before pessary insertion, three measurements were taken: the vaginal apex and the anterior and posterior vaginal walls. In all patients, the results corresponded to stage 3 or higher.

Follow-up was carried out little after pessary insertion and approximately 6 months after the intervention and the feeling of fullness or pressure in the vagina, as well as associated symptoms, were improved significantly (P-value < 0.001), in all points except pb (perineal body) and tvl (total vaginal length) points (Figure 1).

After approximately 6 months, the vaginal discharge was significantly increased, while the feeling of fullness in the vagina was significantly decreased ( $P$-value $<0.001)$.

The results of our study showed that sexual dissatisfaction in patients and their partners, the feeling of incomplete evacuation, fecal and urinary incontinence, frequent urination, and pain or discomfort in the genital region were not significantly different after using a pessary Table 2.

After approximately 6 months, $96.7 \%$ of the women with a successful pessary fitting trial were satisfied and had significance symptoms improvement Tables 3, 4 .

\section{Discussion}

Pelvic organ prolapse is a common complaint in postmenopausal and older women in the entire world [15].

Table 1: Patient Characteristics (number= 68)

$\begin{array}{ll}\text { Age (y): mean(SD) } & \mathbf{6 8 . 9 8 ( 1 0 . 1 5 4 )} \\ \text { Menopause, } \mathbf{n}(\%) & 97.1 \% \\ \text { Previous hysterectomy (percent) } & 10.3 \% \\ \text { Previous prolapse surgery (percent) } & 8.8 \% \\ \text { Sexually active } & 42.6 \% \\ \text { Tendency to surgery before pessary } & 12.1 \%\end{array}$

In particular situations, such as the presence of severe medical comorbidities that make the patient a poor surgical candidate, the preference for nonsurgical treatment or the need to delay surgery for several weeks or months, patients suffering from recurrent pelvic organ prolapse or stress urinary incontinence are recommended to use a vaginal pessary.

The use of a pessary, although having many benefits, also has a few disadvantages that limit its use in patients with local infections. Also, sexually active women who are unable to remove and reinsert the pessary are not appropriate candidates for pessary insertion the most important advantages and disadvantages of pessary mentioned in Table 5.

There are several studies proving that vaginal pessary improves some of the symptoms of pelvic organ prolapse [16].

In a prospective study that Clemons et al. carried out in 2004,17.100 women with symptomatic pelvic organ prolapse were fitted with a pessary, and 73 women had a successful 2-week pessary fitting trial. After approximately 6 months, $92 \%$ of women with a successful pessary fitting trial were satisfied. Dissatisfaction in $8 \%$ of patients was because of occult stress incontinence. Nearly all symptoms resolved; $50 \%$ of the urinary symptoms improved, stress incontinence improved in $45 \%$ of patients and was a common side effect in other patients.

However, in Clemons' study, unlike our study, a valid questionnaire was not used to assess other symptoms like bowel and bladder symptoms. After evaluating the questionnaire, we found out that vaginal discharge and the feeling of fullness in the vagina were significantly decreased at approximately 6 months after the intervention, but other symptoms like urinary incontinence did not change significantly. It is essential to have a proper questionnaire to evaluate the symptoms before and after pessary insertion.

Table 2: Baseline symptoms and change of symptoms after insertion of vaginal pessaries at approximately 6 months.

\begin{tabular}{|c|c|c|c|c|}
\hline \multirow[t]{2}{*}{ Symptom } & \multicolumn{2}{|c|}{ Before pessary } & \multicolumn{2}{|c|}{ Change of symptoms } \\
\hline & & Better & Worse or New created & No change \\
\hline Feeling of fullness in the vagina & $94.9 \%$ & $97.6 \%$ & $0 \%$ & $2.4 \%$ \\
\hline Vaginal soreness and discharge & $8.6 \%$ & $0 \%$ & $50 \%$ & $50 \%$ \\
\hline Urge bowel incontinence & $12.7 \%$ & $10 \%$ & $5 \%$ & $85 \%$ \\
\hline Incomplete bowel evacuation & $19 \%$ & $13.6 \%$ & $4.5 \%$ & $77.3 \%$ \\
\hline Stress urinary incontinence & $34.4 \%$ & $50 \%$ & $3.1 \%$ & $46.9 \%$ \\
\hline Urge urinary incontinence & $53.3 \%$ & $36.1 \%$ & $8.3 \%$ & $55.6 \%$ \\
\hline Incomplete bladder emptying & $35.6 \%$ & $42.4 \%$ & $15.2 \%$ & $42.4 \%$ \\
\hline Pain & $3.4 \%$ & $0 \%$ & $28 \%$ & $72 \%$ \\
\hline Lack of sexual satisfaction & $12.5 \%$ & $27.3 \%$ & $9.1 \%$ & $63.6 \%$ \\
\hline
\end{tabular}


Table 3: Satisfaction after pessary use

\begin{tabular}{llcc}
\multicolumn{2}{l}{ Satisfaction } & Frequency & Percent \\
Valid & Very satisfied & 50 & 82.0 \\
& Satisfied & 5 & 8.2 \\
& No change & 5 & 8.2 \\
& Dissatisfied & 1 & 1.6 \\
Total & & 61 & 100.0
\end{tabular}

Table 4: Symptom improvement after pessary insertion at approximately 6 months

$\begin{array}{llcc}\text { Symptom improvement } & \text { Frequency } & \text { Percent } \\ \text { Valid } & \text { So much better } & 50 & 82.0 \\ & \text { Much better } & 3 & 4.9 \\ & \text { Better } & 2 & 3.3 \\ & \text { No change } & 5 & 8.2 \\ & \text { So much worse } & 1 & 1.6 \\ & \text { Total } & 57 & 93.4 \\ \text { Total } & & 61 & 100.0\end{array}$

Table 5: POP-Q test before and after pessary insertion

$\begin{array}{llrrrr} & & \text { Mean } & \text { N } & \begin{array}{c}\text { Std. } \\ \text { Deviation }\end{array} & \text { P-value } \\ \text { Pair 1 } & \text { Aa_0 } & 1.800 & 60 & 2.0630 & .000 \\ & \text { Aa_1 } & -1.225 & 60 & 1.4451 & \\ \text { Pair 2 } & \text { Ba_0 } & 3.617 & 60 & 2.8394 & .000 \\ & \text { Ba_1 } & -1.050 & 60 & 1.5642 & \\ \text { Pair 3 } & \text { C_0 } & 3.900 & 60 & 3.5052 & .000 \\ & \text { C_1 } & -3.592 & 60 & 2.8351 & \\ \text { Pair 4 } & \text { GH_0 } & 5.983 & 59 & 5.0026 & .011 \\ & \text { GH_1 } & 4.186 & 59 & 1.4707 & \\ \text { Pair 5 } & \text { Pb_0 } & 3.458 & 59 & .9882 & .093 \\ & \text { Pb_1 } & 3.653 & 59 & 1.1790 & \\ \text { Pair 6 } & \text { TVL_0 } & 8.780 & 59 & 1.6302 & .477 \\ & \text { TVL_1 } & 8.932 & 59 & 1.5769 & \\ \text { Pair 7 } & \text { Ap_0 } & -.133 & 60 & 2.1110 & .001 \\ & \text { Ap_1 } & -1.283 & 60 & 1.4391 & \\ \text { Pair 8 } & \text { Bp_0 } & .642 & 60 & 2.8509 & .000 \\ & \text { Bp_1 } & -1.075 & 60 & 1.5940 & \\ \text { Pair 9 } & \text { D_0 } & .690 & 51 & 6.9379 & .000 \\ & \text { D_1 } & -3.912 & 51 & 2.6262 & \\ & & & & & \end{array}$

In another prospective observational study conducted in 2016, 18 women with pelvic organ prolapse presenting for a vaginal pessary fitting were asked to complete the ICIQ-VS questionnaire (International Consultation on Incontinence Modular Questionnaire-Vaginal Symptoms), and their satisfaction was evaluated using the visual

\section{Satisfaction form of Patients with pelvic organ \\ Prolapse \\ Advantages: \\ - Reversible \\ - Having no serious complication \\ - At any time can be removed in case of dissatisfaction \\ - Whenever a patient wants, can remove it and get \\ surgery \\ - Alternative procedure instead of surgery and have no surgical problems \\ Disadvantages: \\ - The need for follow up in the specified intervals for the patient's and frequent examination \\ - Urinary incontinence following pessary insertion}

analogue scale (VAS) prior to pessary fitting, and after 3 and 6 months of treatment. Forty women entered this study, and all vaginal symptoms and the quality of life scores significantly improved after 3 and 6 months of treatment. The use of a vaginal pessary for up to 6 months improved the vaginal symptoms, quality of life and satisfaction in women with pelvic organ prolapse.

Although some patients had some complications like the erosion of the vaginal wall or vaginal discharge, these complications were improved by adequate lubrication and vaginal estrogen treatment.

One strength of this study was that patients were treated with pessaries over 6 months, and followed-up for 3 and 6 months after pessary insertion.

However, in this study, a validated Thai version of the ICIQ-VS and a simple VAS tool were used to interpret patient's satisfaction, while we used the Patient Global Impression of Improvement (PGI-I) questionnaire to assess satisfaction regarding pessary insertion. Also, the stage of pelvic organ prolapse was assessed by a single examiner that performed the POP-Q exams.

One of the weaknesses of this study, unlike our study, was its small sample size.

Similar studies have shown that most women with pelvic organ prolapse tend to use a pessary as a noninvasive treatment $[19,20]$.

Pelvic organs prolapse is one of the most common complaints in older women [21].

Due to the risk of surgery in patients, especially in the elderly, our first recommendation is pessary treatment. Our study showed that pessaries improve POP in the majority of patients and can replace surgery in some patients, especially elderly patients.

The pessary should be noted in patients with many contraindications and high risk of surgery. Also, the pessary is an appropriate treatment option in patients with a history of medical problems such as heart disease, high blood pressure, cerebrovascular disease, and history of myocardial infarction. 
Also, further studies are recommended to focus on other prolapse symptoms with different types of pessaries in order to assess the response to treatment.

\section{Acknowledgment}

We wish to thank Miss Mahnaz Alizadeh and colleagues at the Clinical Research and Development Unit | of Fatemieh Hospital of Hamadan who provided insight and expertise that greatly assisted the research. We also gratefully acknowledge Mrs. Lotfi and the professors of the Pelvic Floor clinic of Imam Khomeini Hospital in Tehran that helped with the manuscript.

\section{Conflict of interest}

The authors declare there is no conflict of interest.

\section{References}

1. Lowder JL, Ghetti C, Nikolajski C, Oliphant SS, Zyczynski HM. Body image perceptions in women with pelvic organ prolapse: a qualitative study. Am J Obstet Gynecol. 2011; 204(5):441-5.

2. Stepp KJ, Walters MD. Urogynecology and Reconstructive Surgery. $3^{\text {th }}$ ed. Philadelphia: Mosby; 2007.

3. DeLancey JO. Anatomic aspects of vaginal eversion after hysterectomy. Am J Obstet Gynecol. 1992; 166(6):1717-24.

4. Jelovsek JE, Maher C, Barber MD. Pelvic organ prolapse. Lancet. 2007; 369(9566):1027-38.

5. Vergeldt TF, Weemhoff M, IntHout J, Kluivers KB. Risk factors for pelvic organ prolapse and its recurrence: a systematic review. Int Urogynecol J. 2015; 26(11):1559-73.

6. Abdool Z, Thakar R, Sultan AH. Prospective evaluation of outcome of vaginal pessaries versus surgery in women with symptomatic pelvic organ prolapse. Int Urogynecol J. 2011; 22(3):273-8.

7. Jones KA, Harmanli O. Pessary use in pelvic organ prolapse and urinary incontinence. Rev Obstet Gynecol. 2010; 3(1):3-9.
8. Handa VL, Jones M. Do pessaries prevent the progression of pelvic organ prolapse? Int Urogynecol J Pelvic Floor Dysfunct. 2002; 13(6):349-51.

9. Clemons JL, Aguilar VC, Tillinghast TA, Jackson ND, Myers DL. Risk factors associated with an unsuccessful pessary fitting trial in women with pelvic organ prolapse. Am J Obstet Gynecol. 2004; 190(2):345-50.

10. Donnelly MJ, Powell-Morgan S, Olsen AL, Nygaard IE. Vaginal pessaries for the management of stress and mixed urinary incontinence. Int Urogynecol J Pelvic Floor Dysfunct. 2004; 15(5):302-7.

11. Powers K, Lazarou G, Wang A, LaCombe J, Bensinger G, Greston WM, et al. Pessary use in advanced pelvic organ prolapse. Int Urogynecol J Pelvic Floor Dysfunct. 2006; 17(2):160-4.

12. Kapoor DS, Thakar R, Sultan AH, Oliver R. Conservative versus surgical management of prolapse: what dictates patient choice? Int Urogynecol J Pelvic Floor Dysfunct. 2009; 20(10):1157-61.

13. Brown LK, Fenner DE, DeLancey JO, Schimpf MO. Defining Patient Knowledge and Perceptions of Vaginal Pessaries for Prolapse and Incontinence. Female Pelvic Med Reconstr Surg. 2016; 22(2):93-7.

14. Yalcin I, Bump R. Validation of two global impression questionnaires for incontinence. Am J Obstet Gynecol. 2003; 189(1):98-101.

15. Woodman PJ, Swift SE, O'Boyle AL, Valley MT, Bland DR, Kahn MA, et al. Prevalence of severe pelvic organ prolapse in relation to job description and socioeconomic status: a multicenter cross sectional study. Int Urogynecol J Pelvic Floor Dysfunct. 2006; 17(4):340-5.

16. Patel M, Mellen C, O'Sullivan DM, LaSala CA. Impact of pessary use on prolapse symptoms, quality of life, and body image. Am J Obstet Gynecol. 2010; 202(5):499.

17. Clemons JL, Aguilar VC, Tillinghast TA, Jackson ND, Myers DL. Patient satisfaction and changes in prolapse and urinary symptoms in women who were fitted successfully with a pessary for pelvic organ prolapse. Am J Obstet Gynecol. 2004; 190(4):1025-9.

18. Anantawat T, Manonai J, Wattanayingcharoenchai $R$, Sarit-apirak S. Impact of a vaginal pessary on the quality of life in women with pelvic organ prolapse. Asian Biomedicine. 2016; 10(3):249-252.

19. Tenfelde S, Tell D, Thomas TN, Kenton K. Quality of life in women who use pessaries for longer than 12 months. Female Pelvic Med Reconstr Surg. 2015; 21(3): 146-9.

20. Mutone MF, Terry C, Hale DS, Benson JT. Factors which influence the short-term success of pessary management of pelvic organ prolapse. Am J Obstet Gynecol 2005; 193(1):89-94.

21. Sitavarin $S$, Wattanayingcharoenchai $R$, Manonai J, Sarit-apirak $S$, Chittacharoen $A$. The characteristics and satisfaction of the patients using vaginal pessaries. J Med Assoc Thai. 2009; 92:744-7.

22. Hullfish KL, Trowbridge ER, Stukenborg GJ. Treatment strategies for pelvic organ prolapse: a cost-effectiveness analysis. Int Urogynecol J. 2011; 22(5):507-15. 